\title{
Controlling Gigahertz and Terahertz Surface Electromagnetic Waves with Metamaterial Resonators
}

\author{
W.-C. Chen, ${ }^{1}$ J. J. Mock, ${ }^{2}$ D. R. Smith, ${ }^{2}$ T. Akalin,,${ }^{3 *}$ and W. J. Padilla ${ }^{1, \dagger}$ \\ ${ }^{1}$ Department of Physics, Boston College, 140 Commonwealth Avenue, Chestnut Hill, Massachusetts 02467, USA \\ ${ }^{2}$ Department of Electrical and Computer Engineering, Duke University, Durham, North Carolina 27708, USA \\ ${ }^{3}$ Institute of Electronics, Microelectronics and Nanotechnology, IEMN UMR CNRS 8520, Lille 1 University, France
}

(Received 6 August 2011; published 6 December 2011)

\begin{abstract}
We computationally and experimentally investigate the use of metamaterial resonators as bandpass filters and other components that enable control of guided surface electromagnetic waves. The guided surface electromagnetic wave propagates on a planar Goubau line, launched via a coplanar waveguide coupler with $50 \Omega$ impedance. Experimental samples targeted for either microwave or terahertz frequencies are measured and shown to be in excellent agreement with simulations. Metamaterial elements are designed to absorb energy only of the planar Goubau line and yield narrow-band resonances with relatively high quality factors. Two independent configurations of coupled metamaterial elements are demonstrated that modify the otherwise flat transmission spectrum of the planar Goubau line. By physically shunting the capacitive gaps of the coupled metamaterial elements, we demonstrate the potential for a large dynamic range in transmissivity, suggesting the use of this configuration for highbandwidth terahertz communications.
\end{abstract}

DOI: 10.1103/PhysRevX.1.021016

Surface electromagnetic waves are propagating solutions to Maxwell's equations in which the electromagnetic energy is bound to an interface between two different media and travels without radiation loss [1,2]. Common examples are the Sommerfeld wave, a radial cylindrical wave [3-6], and the Zenneck wave [7,8], all of which are bound to a conducting interface and propagate without radiation. Early on, various methods were developed that enhanced coupling of electromagnetic waves to planar conducting surfaces, such as the use of dielectric coatings and surface corrugation $[1,9,10]$, which can produce strong confinement even when the supporting metal is assumed to have an infinite conductivity.

Guided waves are of vital importance in routing electromagnetic energy from a source to a device or system and eventually to a receiver. Dielectric guides, hollow waveguides, and two-conductor transmission lines (e.g., coaxial) are typical examples and operate with extreme efficiency at very low frequencies $(<1 \mathrm{GH} z)$ [11]. Lumped components-such as packaged capacitors and inductors - can be implemented easily to form a wide variety of filter networks and other components that can be integrated into the line. However, the availability of packaged circuit components that operate at high frequencies is severely limited and, where they exist, such

\footnotetext{
*Tahsin.Akalin@iemn.univ-lille1.fr
}

${ }^{\dagger}$ Willie.Padilla@bc.edu

Published by the American Physical Society under the terms of the Creative Commons Attribution 3.0 License. Further distribution of this work must maintain attribution to the author(s) and the published article's title, journal citation, and DOI.
Subject Areas: Metamaterials, Optoelectronics, Plasmonics

components tend to be rare and are generally expensive. At higher frequencies, the losses associated with conventional radio frequency cables and microstrip lines tend to rise, making them less suitable for integrated devices. At these higher frequencies, the properties of surface-electromagnetic-wave (SEW) devices become more favorable, making them competitive for integrateddevice applications, especially at terahertz and higher frequencies.

Although the initial motivation for metamaterials was the ability to achieve a negative index of refraction $[12,13]$, over the past several years, metamaterial elements have been investigated for use as filters and other components in transmission-line structures. There have been some works utilizing the so-called split-ring resonator (SRR) [14], to couple with the magnetic component of light within a waveguide, or the complementary split-ring resonator (CSRR) [15-21], for electric coupling on a coplanarwaveguide (CPW) structure. In these systems, metamaterials serve as parasitic elements producing stopbands $[15,16]$ or passbands $[14,17]$. Other works have demonstrated metamaterials (MMs) capable of more sophisticated responses, such as nonreciprocity [18] and dynamic tunability [22-24]. All of these works utilize metamaterials to couple to conventional transmission systems in which the electromagnetic energy is effectively confined to a region of space by means of two or more conducting structures: coaxial, coplanar, stripline, slotline, etc. However, under certain conditions, other types of transmission may exist in which the energy is not rigidly confined but rather is bound to a single surface or structure and propagates without radiation - the surface electromagnetic wave [25]. 
Here, we report on computational and experimental investigations of the control of SEWs with novel metamaterial elements and detail several examples. As a demonstration of the flexibility and scalability of the designs, we show results over two bands of the electromagnetic spectrum - gigahertz $(\mathrm{GHz})$ and terahertz $(\mathrm{THz})$ frequencies. For guiding our electromagnetic waves, we chose a single planar wire transmission line that lies on top of a supporting dielectric substrate, known as a planar Goubau line (PGL) - previously discussed in detail elsewhere [26,27]. Geometry of the PGL is optimized in order to obtain a broadband electromagnetic spectrum in both frequency ranges studied.

The PGL and metamaterial structures are simulated in the time domain with a commercial full-wave electromagnetic mode-transient solver. For the simulations, we use metallic conductivities with values of $\sigma_{0}=4.7 \times 10^{7}(\mathrm{~S} / \mathrm{m})$ and $\sigma_{0}=4.56 \times 10^{7}(\mathrm{~S} / \mathrm{m})$, and the underlying substrates are simulated as lossy dielectrics with values of $\tilde{\boldsymbol{\epsilon}}=3.75+$ $0.025 i$ and $\tilde{\boldsymbol{\epsilon}}=2.828+0.056 i$ for the $\mathrm{GHz}$ and $\mathrm{THz}$ materials, respectively. The $\mathrm{GHz}$ metallic structures have thicknesses of $17 \mu \mathrm{m}$, and supporting substrates are $200 \mu \mathrm{m}$ thick. In the $\mathrm{THz}$ regime, all metallic elements are $200 \mathrm{~nm}$ thick with a substrate thickness of $350 \mu \mathrm{m}$. The cross section (width $\times$ height) of the GHz PGL is $150 \mu \mathrm{m} \times 17 \mu \mathrm{m}$, while that of the THz PGL is $4 \mu \mathrm{m} \times$ $0.2 \mu \mathrm{m}$. The total lengths are $320 \mathrm{~mm}$ and $5000 \mu \mathrm{m}$ for the GHz and THz PGLs, respectively.

The PGL supports an approximately axial cylindrical SEW. The insets in Fig. 1(a) show a PGL in cross section, centered in each plot, with the supporting substrate shown underneath. Plotted in the insets in Fig. 1(a) are the electric (left) and magnetic (right) field vectors. The modes are transverse-electric-magnetic-like (TEM-like), with the electric field directed uniformly radially outward and the magnetic field circulating clockwise around the wire. The red curves in Fig. 1(a) show the spatial dependence of both the electric-field strength (left) and magnetic-flux density (right) beginning from the center of the wire and moving horizontally outward. Both fields fall off approximately exponentially [28].

In order to couple electromagnetic energy into and out of the PGL, we utilize a coplanar-waveguide (CPW) section

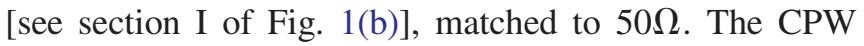
transitions via section II to a two-dimensional (2D), planar horn-shaped antenna (section III), where the center conductor of the PGL line lies in the middle. The parameters that maximize the transfer of radiation (and the total length of the PGL itself) are (in millimeters) $L=320, L 1=20, L 2=$ 7.5 , and $R=20$ for the GHz PGL; and (in microns) $L=$ $5000, L 1=300, L 2=300$, and $R=800$ for the THz PGL. In Fig. 2(a) we show simulated results for each of the PGLs. Both exhibit a broadband spectrum and usable range of $47 \mathrm{GHz}$ and $384 \mathrm{GHz}$ for the $\mathrm{GHz}$ and THz PGLs, respectively, where the usable range is defined as the bandwidth at roughly $1 \%$ of the peak value on both ends. Figure 2 (b)
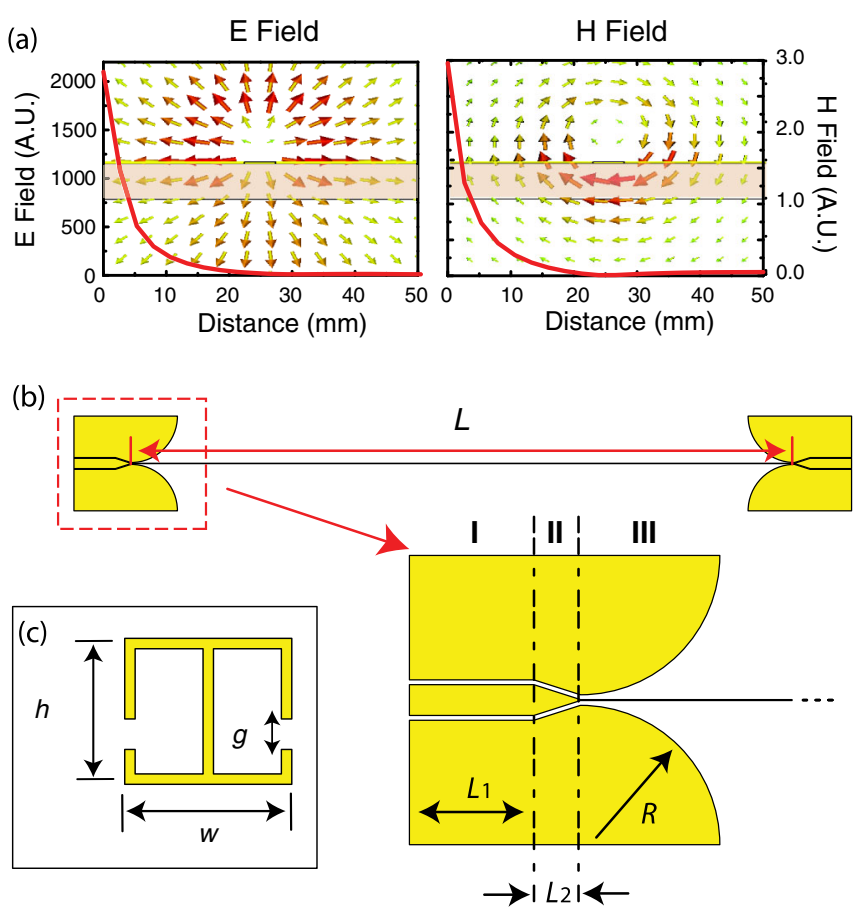

FIG. 1. Simulated modes and dimensions of the planar Goubau line, and metamaterial schematic. Left panel of (a) plots the norm of the electric field strength as a function of horizontal distance away from the center of the GHz PGL. Right panel of (a) plots the norm of the magnetic field in the same manner. Vector plots of the electric (left) and magnetic (right) field vectors are shown as the insets of Fig. 1(a). The very small gray rectangle at the center of each inset illustrates the cross section of the PGL. The larger rectangle is a cross sectional view of the supporting substrate. Panels (b) and (c) show the dimensions of the PGL and metamaterial, respectively.

shows an expanded view of the simulated spectra of the $\mathrm{GHz}$ (solid blue curves) and $\mathrm{THz}$ (solid red curves) PGLs.

We seek to couple to the electric field of the PGL using a metamaterial element [see the left inset to Fig. 1(a)]. As a starting point, we begin with the so-called electric-ring resonator (ERR) [29,30] element. The ERR has shown the ability to yield strong interactions with the electric-field component of a free-space TEM wave with good oscillator strength [30]. We optimize dimensions of the ERRs in order to obtain the highest quality factor $(Q)$, defined as $\omega_{0} / \triangle \omega$, where $\omega_{0}$ is the resonant frequency and $\triangle \omega$ is the FWHM of the resonance. The optimized geometry is shown in Fig. 1(c), with the dimensions of $g=0.39, w=2.4$, and $h=2.1$ (in millimeters) for the GHz MMs and $g=6, w=$ 105 , and $h=90$ (in microns) for the THz MMs.

Metamaterials and PGLs were fabricated with printedcircuit-board technology (GHz structures) and optical lithography (THz structures), with dimensions identical to those simulated. The PCB is copper-clad FR4 (a glassreinforced epoxy laminate), and the $\mathrm{THz}$ structures are nanopatterned gold deposited on a thin fused-silica substrate. Masks were used for the fabrication of both 


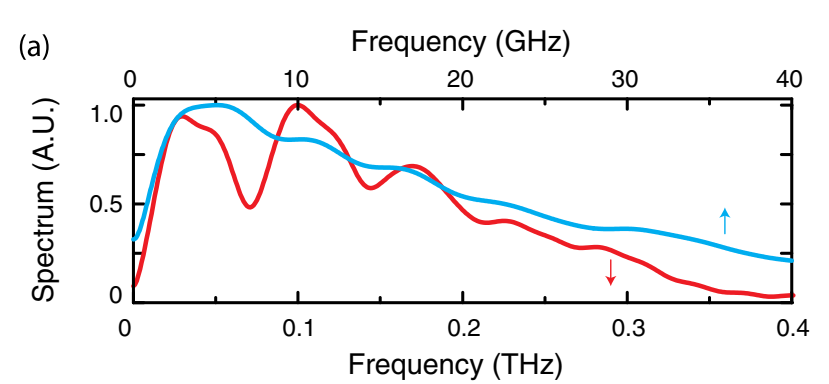

(b) Frequency (GHz)

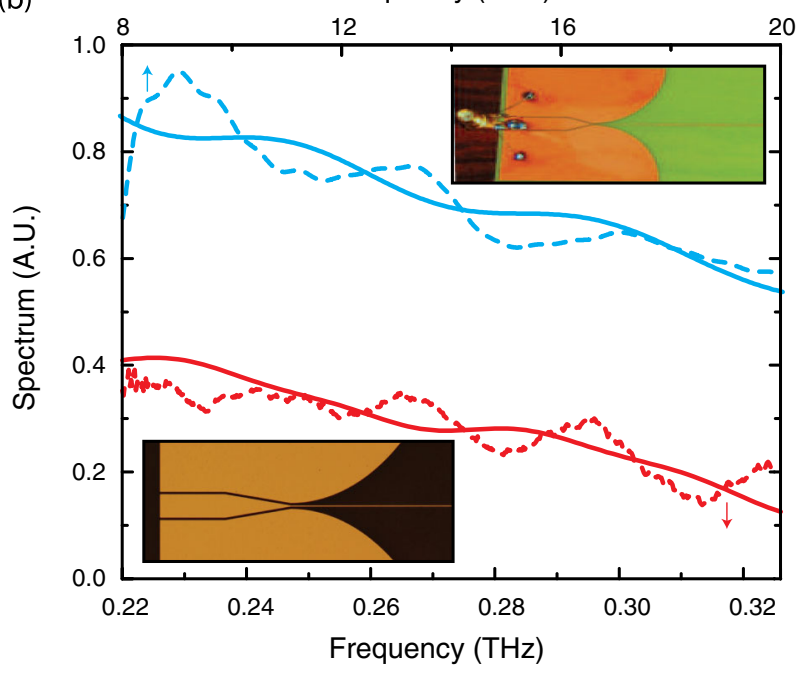

FIG. 2. (a) Simulated spectra of $\mathrm{GHz}$ (blue curve) and $\mathrm{THz}$ (red curve) PGL waveguides. (b) Experimental (dashed curves) and simulated (solid curves) spectra for both the $\mathrm{GHz}$ (blue) and $\mathrm{THz}$ (red) PGLs. Insets are optical images of the gigahertz (top right) and terahertz (bottom left) waveguides. The blue and red arrows in both panels indicate the $\mathrm{x}$ axes of the individual curves.

structures. Photographs of the fabricated PGLs are shown as the insets to Fig. 2(b), and photos of the THz metamaterial/ PGL structures are shown as the insets to Figs. 3(a) and 3(b). Metamaterial resonators are placed in the middle of the planar Goubau line section and on both sides of the line. The transverse distance between the PGL and the resonators is $1.6 \mathrm{~mm}$ for the $\mathrm{GHz}$ design, and 30 microns for the $\mathrm{THz}$ one. For the case where two different metamaterial resonators are used, the distance between them is 300 microns for the $\mathrm{THz}$ design.

All structures were characterized with vector network analyzers (VNAs) operating separately in the gigahertz band from 8 to $20 \mathrm{GHz}$ and in the terahertz band from 0.22 to $0.325 \mathrm{THz}$. The complex frequency-dependent scattering parameters- the transmission coefficient $\left(\mathrm{S}_{21}\right)$ and the reflection coefficient $\left(\mathrm{S}_{11}\right)$-were characterized for the PGLs with and without metamaterials. All spectra were normalized with respect to a PGL with no metamaterial elements.

The experimental spectra of the PGL, shown in Fig. 2(b) as the dashed blue $(\mathrm{GHz})$ and dashed red $(\mathrm{THz})$ lines, are in good general agreement with simulated spectra (the solid
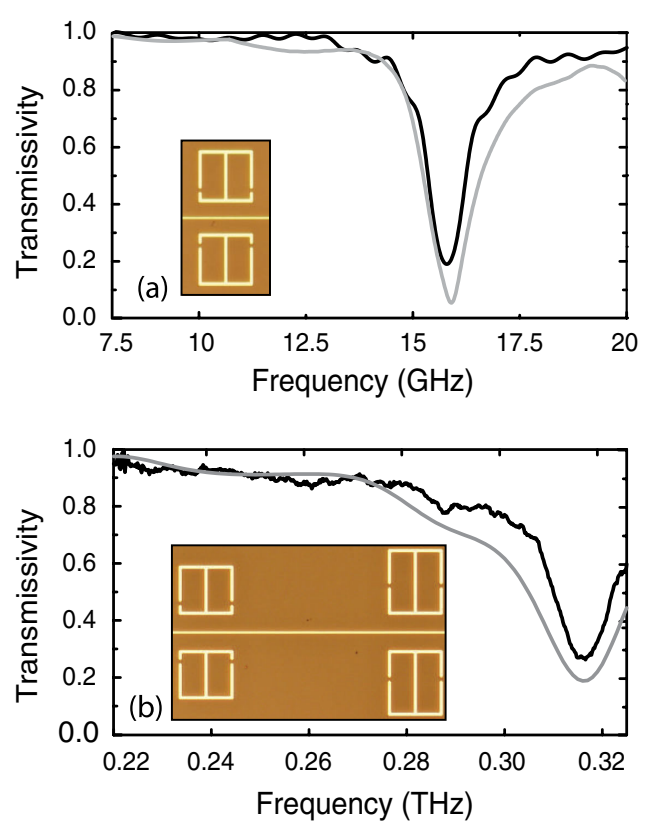

FIG. 3. Simulated (gray curves) and experimental (black curves) transmissivity of (a) the GHz MMs and (b) the THz MMs on the PGL. The two insets are optical images of single-pair (top) and dual-pair (bottom) $\mathrm{THz}$ metamaterial elements adjacent to the PGL.

curves). Here, impedances due to external connections to the PGL (which are not taken into account in simulation) lead to small oscillations lying on top of the spectra. Having characterized the spectra of each PGL, we now turn toward study of the interaction of metamaterial elements with the broad spectrum provided by the PGL via the transmissivity $\left(T(\omega)=\left|\mathrm{S}_{21}\right|^{2}\right)$. We calculate $T(\omega)$ by dividing the transmitted spectra of the PGL plus metamaterial by the transmitted spectra of the PGL alone. In the gray curves of Figs. 3(a) and 3(b), we show the simulated absolute value $T(\omega)$ of metamaterial elements for both the $\mathrm{GHz}$ and $\mathrm{THz}$ structures, respectively. It is evident that only one prominent minimum is observed on each PGL, and values of $T(\omega)=5 \%$ at $16 \mathrm{GHz}$ and $T(\omega)=21 \%$ at $0.315 \mathrm{THz}$ are obtained [31]. Simulations indicate $Q$ values of 11.4 and 10.5 for the $\mathrm{GHz}$ and $\mathrm{THz}$ metamaterials, respectively. Outside of the resonant region, the PGL achieves over $80 \%$ transmissivity, indicating that metamaterial elements yield only a narrow-band resonance. The normalized experimental transmissivity (black curves) of the $\mathrm{GHz}$ and $\mathrm{THz}$ metamaterial elements are plotted in Figs. 3(a) and 3(b), where we observe good agreement with simulation (gray curves). Consistent with the simulations, we observe resonances around $16 \mathrm{GHz}$ and $0.32 \mathrm{THz}$ (see black curves in Fig. 3). A slightly higher transmitted energy and narrower bandwidth is observed, for both configurations, at the resonance frequency, and in each case the measured $Q$ is higher than that predicted by simulation.

In order to verify absorptivity and coupling of the metamaterial element to the waveguide, we next explore a 


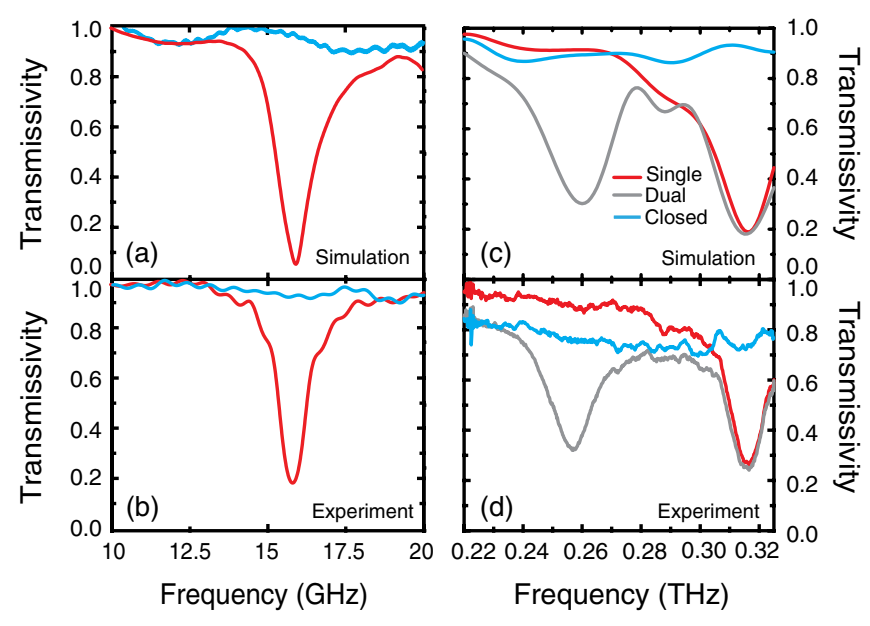

FIG. 4. Simulated (a) and (c) and experimental (b) and (d) results of MMs on the PGL. Panels (a) and (b) are for the single-pair GHZ-PGL case, with the red and blue curves corresponding to the cases of the open-gap and closed-gap resonators, respectively. Panels (c) and (d) show the data for the dual-pair THz-PGL case, plotted as the gray curves and in comparison with the cases of single-pair open-gap (red) and closed-gap (blue) resonators in the $\mathrm{THz}$ regime.

nonresonant metamaterial configuration and study the transmission dependence. We utilize a geometry similar to that previously published [32] — essentially an ERR in which the gaps are closed. This "closed-gap" configuration has been shown to be nonresonant, as the capacitive portion of the metamaterial element has been removed. The blue curve of Fig. 4(a) demonstrates that the simulated closedgap metamaterial elements do not couple to the SEW carried by the PGL, at least within the range characterized. Indeed, the transmissivity is observed to be roughly frequency-independent with values above $90 \%$. For a direct comparison, we replot the simulated $T(\omega)$ for a resonant (open-gap) metamaterial element, shown as the red curve in Fig. 4(a) [33]. The experimental results match well with simulations, shown as the blue (closed gaps) and red (open gaps) curves of Fig. 4(b).

Although we are able to perform experiments only over a narrow range of the large bandwidth carried by the PGL, it should be possible to utilize multiunit cell designs with which one could "encode" more resonances onto the broad spectrum. We fashion a metamaterial which is slightly larger in size than the previously demonstrated element, shown in Fig. 3(b). We simulate two differently sized metamaterial elements [shown as the inset to Fig. 3(b)], which interact with the $\mathrm{THz} \mathrm{SEW}$ - shown as the gray curves of Fig. 4(c). We observe a new resonant absorption in $T(\omega)$ at $0.260 \mathrm{THz}$, while simulations indicate little change to the higher-energy metamaterial absorption at $0.320 \mathrm{THz}$. With a view toward future dynamical or switchable SEW metamaterials, we may again close the gaps of the metamaterial elements. The simulated results in which both resonators are in the nonresonant configuration are

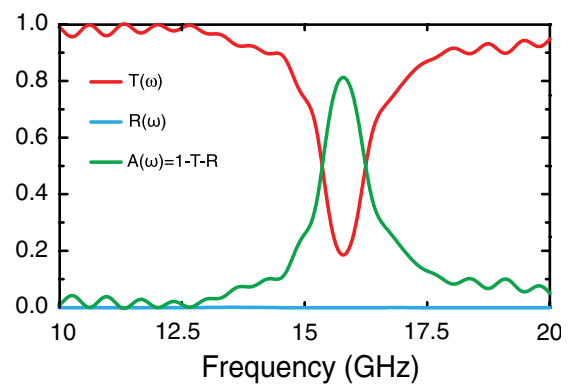

FIG. 5. Experimental results of the reflectivity, transmissivity, and absorptivity of a $\mathrm{GHz}$ metamaterial interacting with the SEW carried by a PGL.

shown as the blue curves of Fig. 4(c). No resonant absorption features are observable, and transmissivity is relatively featureless, with values of roughly $T(\omega)=0.9$. To compare responses between the single-pair and the dual-pair metamaterial elements on the PGL, we replot the transmissivity for the single metamaterial element with the gaps open, shown as the red curves of Fig. 4(c). As can be observed, there is little change in the higher-lying resonance-it is essentially unaffected. Figure 4(d) shows experimental measurements where both ERR elements are resonant (gray curves), both ERRs are short-circuited (blue curves), and where only a single metamaterial is resonant (red curve). A comparison of the simulated and experimental curves of Fig. 4 shows relatively good agreement in all cases.

We now highlight several salient features of the metamaterial designs presented here. A notable difference between free-space and surface-wave metamaterials is that, for the latter, we need only two elements instead of an array of elements in proximity to the PGL. Indeed, we still realize, with the two elements, substantial electromagnetic response signified by large dips in the transmission. This is a bit surprising considering the small cross section that the metamaterial elements occupy compared to the extent and homogeneity of the mode (see the plot of the electric field in the left inset to Fig. 1(a)). However, we must recall that the electric and magnetic fields fall off exponentially as a function of distance from the center of the line. Thus, so long as the elements are spaced relatively close to the PGL, the resonant dips yield relatively large $Q \mathrm{~s}$ with experimental values of $Q=14.9$ and $Q=18.4$ at $\mathrm{GHz}$ and $\mathrm{THz}$ frequencies, respectively. In contrast, the nonresonant metamaterial-element configuration attains relatively large frequency-independent values for $T(\omega)$. Thus, the resonant metamaterial elements are responsible for the lack of transmission at the resonance frequency. Obviously, energy in the broadband carried by the PGL which is not transmitted can be reflected, absorbed, or scattered by the metamaterial element. In Fig. 5, we present experimental results for $\mathrm{GHz}$ structures which reveal that energy is primarily absorbed by the metamaterial. As can be observed, the reflectivity (blue line of Fig. 5), is essentially zero across the entire band, 
while the transmissivity achieves a minimum of approximately $20 \%$. This means that the metamaterial elements do not significantly modify the impedance of the PGL. Scattered radiation due to the metamaterial elements has been also studied in simulation (not shown), and it was found to be negligible across the range shown in Fig. 5. Thus, the energy which is not transmitted is absorbed by the metamaterial elements, as shown by the green curves of Fig. 5, with values reaching 80\%. As shown in Figs. 4(c) and 4(d), a second absorptive dip may be encoded onto the broad spectrum of the PGL via a different metamaterial element, with similar electromagnetic properties, i.e., strong absorption characteristics, like that of a single metamaterial element.

The strong absorptivity achieved by the metamaterial elements, in comparison to the high transmissivity achieved by the nonresonant metamaterial elements, suggests dynamical applications. For example, energy absorbed within the metamaterial elements is focused within the capacitive gaps of the structures. Thus, utilization of field-effect transistors or semiconductors placed within the gaps of the metamaterials would provide the ability to modulate the amplitude and frequency location of the resonance via electronic or optical means. Further, as shown in Fig. 4, these metamaterial resonances may be modulated independently. By adding more elements along the length of the PGL, it may thus be possible to encode a large amount of information on the broad electromagnetic spectrum, which would enable high-bandwidth $\mathrm{THz}$ communication.

The planar Goubau lines explored here have been demonstrated to carry a wide-band surface-electromagneticwave spectrum. Newly designed metamaterial elements have been shown to strongly interact with the particular mode exhibited by the PGL SEW and to yield high- $Q$ resonances. Multiple metamaterial element resonances have been coded onto the broad PGL spectrum, and independent shunting of each metamaterial resonance has been demonstrated.

This project has been supported in part by the DOD/ Office of Naval Research under Contract No. N00014-071-0819 and NSF under Contract No. ECCS-1002340.

[1] A.F. Harvey, Periodic and Guiding Structures at Microwave Frequencies, IEEE Trans. Microw. Theory Tech. 8, 30 (1960).

[2] J. O'Hara, R. D. Averitt, and A. J. Taylor, Prism Coupling to Terahertz Surface Plasmon Polaritons, Opt. Express 13, 6117 (2005).

[3] G. Goubau, Surface Waves and Their Application to Transmission Lines, J. Appl. Phys. 21, 1119 (1950).

[4] G. Goubau, On the Excitation of Surface Waves, Proc. IRE 40, 865 (1952).

[5] G. Goubau, Open Wire Lines, IEEE Trans. Microw. Theory Tech. 4, 197 (1956).
[6] G. Goubau, Surface-Wave Transmission Line, Acta Tech. Acad. Sci. Hung. 17, 269 (1957).

[7] V.N. Datsko and A. A. Kopylov, On Surface Electromagnetic Waves, Phys. Uspekhi 51, 101 (2008).

[8] A. V. Kukushkin, On the Existence and Physical Meaning of the Zenneck Wave, Phys. Uspekhi 52, 755 (2009).

[9] R. S. Elliott, On the Theory of Corrugated Plane Surfaces, IRE Trans. Antennas Propag. 2, 71 (1954).

[10] J. B. Pendry, L. Martin-Moreno, and F. J. Garcia-Vidal, Mimicking Surface Plasmons with Structured Surfaces, Science 305, 847 (2004).

[11] S. Ramo, J. R. Whinnery, and T. V. Duzer, Fields and Waves in Communication Electronics (John Wiley \& Sons, New York, 1994), 3rd ed.

[12] D. R. Smith, W. J. Padilla, D. C. Vier, S. C. Nemat-Nasser, and S. Schultz, A Composite Medium with Simultaneously Negative Permeability and Permittivity, Phys. Rev. Lett. 84, 4184 (2000).

[13] R. A. Shelby, D. R. Smith, and S. Schultz, Experimental Verification of a Negative Index of Refraction, Science 292, 77 (2001).

[14] F. Martin, F. Falcone, J. Bonache, R. Marques, and M. Sorolla, Miniaturized Coplanar Waveguide Stop Band Filters Based on Multiple Tuned Split Ring Resonators, Microw. Wirel. Compon. Lett. 13, 511 (2003).

[15] F. Falcone, F. Martin, J. Bonache, R. Marques, and M. Sorolla, Coplanar Waveguide Structures Loaded with Split-Ring Resonators, Microw. Opt. Technol. Lett. 40, 3 (2004).

[16] A. lbraheem and M. Koch, Coplanar Waveguide Metamaterials: The Role of Bandwidth Modifying Slots, Appl. Phys. Lett. 91, 113517 (2007).

[17] I. A. I. Al-Naib, C. Jansen, and M. Koch, Miniaturized Bandpass Filter Based on Metamaterials Resonators: A Conceptual Study, J. Phys. D 41, 205002 (2008).

[18] M. A. Abdalla and Z. Hu, Nonreciprocal Left Handed Coplanar Waveguide over Ferrite Substrate with Only Shunt Inductive Load, Microw. Opt. Technol. Lett. 49, 2810 (2007).

[19] I. A. I. Al-Naib and M. Koch, Highly Miniaturized Single Metal Layer CPW Bandstop Filters Based on Spiral Resonators, Electron. Lett. 46, 1274 (2010).

[20] I. A. I. Al-Naib, C. Jansen, and M. Koch, Single Metal Layer CPW Metamaterial Bandpass Filter, Prog. Electromagn. Res. Lett. 17, 153 (2010).

[21] F. Falcone, T. Lopetegi, M. A. G. Laso, J. D. Baena, J. Bonache, M. Beruete, R. Marques, F. Martin, and M. Sorolla, Babinet Principle Applied to the Design of Metasurfaces and Metamaterials, Phys. Rev. Lett. 93, 197401 (2004).

[22] I. Gil, J. Bonache, J. Garcia-Garcia, and F. Martin, Tunable Metamaterial Transmission Lines Based on Varactor-Loaded Split-Ring Resonators, IEEE Trans. Microw. Theory Tech. 54, 2665 (2006).

[23] A. Velez, J. Bonache, and F. Martin, Effects of Varying the Series Capacitance in CSRR-Loaded Metamaterials Transmission Lines, Microw. Opt. Technol. Lett. 49, 2245 (2007).

[24] E. Ekmekci, Kagan Topalli, Tayfun Akin, and G. TurhanSayan, A Tunable Multi-Band Metamaterial Design Using 
Micro-Split SRR Structures, Opt. Express 17, 16046 (2009).

[25] J. Brown, The Types of Wave Which May Exist near a Guiding Surface, Proc. IEEE 100, 363 (1953).

[26] A. Treizebre, T. Akalin, and B. Bocquet, Planar Excitation of Goubau Transmission Lines for THz BioMEMS, Microw. Wirel. Compon. Lett. 15, 886 (2005).

[27] T. Akalin, A. Treizebre, and B. Bocquet, Single-Wire Transmission Lines at Terahertz Frequencies, IEEE Trans. Microw. Theor. Tech. 54, 2762 (2006).

[28] See supplemental material at http://link.aps.org/ supplemental/10.1103/PhysRevX.1.021016 for an animation of the electric and the magnetic fields of the SEW propagating on the PGL.

[29] D. Schurig, J. J. Mock, and D. R. Smith, ElectricField-Coupled Resonators for Negative Permittivity Metamaterials, Appl. Phys. Lett. 88, 041109 (2006).
[30] W. J. Padilla, M. T. Aronsson, C. Highstrete, M. Lee, A.J. Taylor, and R.D. Averitt, Electrically Resonant Terahertz Metamaterials: Theoretical and Experimental Investigations, Phys. Rev. B 75, 041102 (2007).

[31] See supplemental material at http://link.aps.org/ supplemental/10.1103/PhysRevX.1.021016 for an animation of the time-varying electric field of the SEW interacting with the gigahertz MM elements at the resonant frequency.

[32] E. N. Economou, T. Koschny, and C. M. Soukoulis, Strong Diamagnetic Response in Split-Ring-Resonator Metamaterials: Numerical Study and Two-Loop Models, Phys. Rev. B 77, 092401 (2008).

[33] See supplemental material at http://link.aps.org/ supplemental/10.1103/PhysRevX.1.021016 for an animation of the time-varying electric fields of the SEW interacting with two independent geometries: resonant and nonresonant MM elements. 